\title{
Ecoescleroterapia com espuma no tratamento da insuficiência venosa crônica*
}

\author{
Ultrasound-guided foam sclerotherapy in the treatment of chronic venous insufficiency \\ Sandro Ceratti ${ }^{1}$, Fabrício Macedo Okano $^{2}$, Alexandre B. Góes Pontes ${ }^{3}$, Antônio Luiz Pontes ${ }^{3}$, \\ Rogério Nastri ${ }^{3}$
}

\begin{abstract}
Resumo Objetivo: Descrever os resultados da ecoescleroterapia com espuma no tratamento de insuficiência venosa crônica. Materiais e Métodos: Série de casos longitudinal. Entre janeiro de 2007 e novembro de 2009, 18 pacientes com insuficiência venosa crônica foram tratados com ecoescleroterapia com espuma em uma clínica particular. 0 método de Tessari foi utilizado para produção da espuma e o seguimento dos pacientes variou de 4 a 44 meses. Os desfechos primários foram: oclusão total da veia tratada e reepitelização total das úlceras. Os desfechos secundários foram: recanalização parcial com redução do calibre da veia, melhora estética e satisfação do paciente. Resultados: A taxa de oclusão total ou recanalização parcial foi de $83,4 \%$ na primeira revisão $(55,6 \%$ de oclusão total e $27,8 \%$ de recanalização parcial) e de $66 \%$ (44,4\% de oclusão total e 22,2\% de recanalização parcial) entre os pacientes que tiveram uma segunda revisão. A taxa de remissão de varizes foi de $80 \%$ e a taxa de cura das úlceras foi de $70 \%$. Conclusão: A ecoescleroterapia mostrou-se um procedimento seguro e eficaz para o tratamento de insuficiência venosa crônica nesse grupo de pacientes. As complicações obsenadas foram mínimas e a maioria dos pacientes referiu satisfação com os resultados do tratamento.
\end{abstract}

Unitermos: Úlcera varicosa; Varizes; Insuficiência venosa; Escleroterapia.

\begin{abstract}
Objective: To describe the results of foam sclerotherapy in the treatment of chronic venous insufficiency. Materials and Methods: Longitudinal study of cases developed in the period between January of 2007 and November of 2009, with 18 patients with venous insufficiency treated with ultrasound-guided foam sclerotherapy in a private clinic. The Tessari's method was utilized to produce the foam and the length of the patients' follow-up varied from 4 to 44 months. Total occlusion of the treated vein and complete ulcer reepithelialization were the primary outcomes, and the secondary ones were vein caliber reduction, aesthetic improvement and patient's satisfaction. Results: The rate of total or partial occlusion achieved $83.4 \%$ in the first review (55.6\% total and $27.8 \%$ partial) and $66 \%(44.4 \%$ total and $22.2 \%$ partial) among the patients who underwent a second review. The rate of varicose veins occlusion achieved $80 \%$ and the rate of ulcer healing achieved $70 \%$. Conclusion: Ultrasound-guided sclerotherapy has demonstrated to be a safe and effective procedure for the management of chronic venous insufficiency in this group of patients. The incidence of complications was minimal and most of the patients reported satisfaction with the results of the treatment.
\end{abstract}

Keywords: Varicose ulcer; Varicose veins; Venous insufficiency; Sclerotherapy.

Ceratti S, Okano FM, Pontes ABG, Pontes AL, Nastri R. Ecoescleroterapia com espuma no tratamento da insuficiência venosa crônica. Radiol Bras. 2011 Mai/Jun;44(3):167-171.

\section{INTRODUÇÃO}

Escleroterapia é a eliminação de veias varicosas por meio da aplicação de uma substância esclerosante no lúmen da veia ${ }^{(\mathbf{1})}$.

* Trabalho realizado na RadMed - Radiologia e Serviços Mé dicos SS, São Roque, SP, Brasil.

1. Membro Titular do Colégio Brasileiro de Radiologia e Diagnóstico por Imagem (CBR), Médico Assistente do Conjunto Hospitalar de Sorocaba, Professor Convidado da Faculdade de Medicina da Pontifícia Universidade Católica de São Paulo (PUCSP), Médico Radiologista da RadMed - Radiologia e Senviços Médicos SS, São Roque, SP, Brasil.

2. Cirurgião Vascular, Diretor Clínico da Unimed São Roque, São Roque, SP, Brasil.

3. Membros Titulares do Colégio Brasileirto de Radiologia e Diagnóstico por Imagem (CBR), Médicos Radiologistas da RadMed - Radiologia e Serviços Médicos SS, São Roque, SP, Brasil.
A ecoescleroterapia com espuma é a aplicação de um agente esclerosante em forma de espuma, guiada pelo ultrassom, em uma determinada veia insuficiente, com o objetivo de ocluir ou reduzir o diâmetro do vaso $^{(2,3)}$. A aplicação do agente esclerosante, em forma de espuma, provoca o deslocamento do sangue na veia e o contato da espuma com o endotélio ocasiona vasoespasmo e oclusão do vaso ${ }^{(3)}$.

Endereço para correspondência: Dr. Sandro Ceratti. Rua Santana, 142, Vila Marques. São Roque, SP, Brasil, 18130-555. E-mail: sanceratti@gmail.com

Recebido para publicação em 23/9/2010. Aceito, após revisão, em 26/4/2011.
Os agentes esclerosantes causam irritação química da íntima venosa que provoca inflamação da camada endotelial do vaso. Essa inflamação dá origem a um trombo local anexo à parede do vaso e as veias se transformam em um cordão fibroso ou esclerosado $^{(\mathbf{1})}$. Esse processo inflamatório ocorre, pois os agentes esclerosantes contêm um polo hidrofílico e outro hidrofóbico, que agem alterando a tensão superficial das células endoteliais. O polo hidrofóbico liga-se à superfície da célula e o polo hidrofílico atrai água para dentro da célula, resultando em rápida e intensa hidratação celu$\operatorname{lar}^{(\mathbf{1})}$. Os agentes esclerosantes mais usados 
são o polidocanol e tetradecyl sulfato de sódio, em concentrações que variam de $1 \%$ a $3 \%$ e volumes que variam de 2 a $15 \mathrm{ml}^{(3-8)}$.

A utilização da ecoescleroterapia com espuma para tratamento de insuficiência venosa tem ganhado popularidade nos últimos anos ${ }^{(9)}$. Os primeiros relatos do uso de espuma na escleroterapia foram realizados por Orbach, em $1944^{(\mathbf{1 0})}$, que propôs a técnica de bloqueio de ar, que consistia na introdução de ar antes do esclerosante, esvaziando o vaso de sangue para que o esclerosante tivesse maior contato com a parede do vaso. Essa técnica foi usada apenas para veias varicosas pequenas e médias e foi pouco utilizada até que Cabrera desenvolveu sua técnica de microespuma, que incluía a injeção de um gás fisiológico no agente esclerosante guiado pelo ultrassom $^{(11)}$. Os trabalhos de Cabrera permitiram o aprimoramento da ecoescleroterapia, ampliando suas indicações e usos ${ }^{(12,13)}$.

A ecoescleroterapia com espuma é um procedimento seguro e eficaz, com baixo índice de complicações, que tem sido utilizado principalmente para o tratamento de varizes de membros inferiores e que oferece resultados semelhantes aos da cirurgia $^{(2,13,14)}$. Além disso, a ecoescleroterapia é um procedimento relativamente barato, minimamente invasivo e que pode ser repetido várias vezes no caso de recorrência das veias incompetentes ${ }^{(\mathbf{1 5})}$.

Acredita-se que a eficácia, a simplicidade, a economia e as variadas possibilidades de aplicação façam da ecoescleroterapia uma modalidade de tratamento atrativa e eficaz, além de possibilitar o tratamento de pacientes que não tinham acesso ou tinham contraindicações ao procedimento cirúrgico ${ }^{(2,13)}$.

A ecoescleroterapia com espuma ainda é um procedimento pouco divulgado no cenário nacional, embora exista uma vasta literatura internacional sobre o tema, mostrando resultados promissores.

Este estudo teve como objetivo descrever os resultados da ecoescleroterapia com espuma no tratamento da insuficiência venosa crônica.

\section{MATERIAIS E MÉTODOS}

Estudo do tipo série de casos longitudinal. Entre janeiro de 2007 e novembro de
2009, foram tratados 18 pacientes com insuficiência venosa crônica em uma clínica particular. A insuficiência venosa crônica foi definida pela presença de varizes ou úlceras venosas crônicas, relacionadas à incompetência da safena magna. Os critérios de inclusão foram: insuficiência venosa crônica, classificação da gravidade clínica (CEAP - clinical severity, etiology, anatomy, pathophysiology) entre 3 e 6 (a classificação varia de 0 a 6) e contraindicação ao tratamento cirúrgico. Os critérios de exclusão foram: trombose venosa profunda prévia, comunicação interatrial ou interventricular (avaliada pelo ecocardiograma antes do procedimento), insuficiência arterial periférica e processo inflamatório em atividade. Os pacientes receberam informações sobre os objetivos do estudo e assinaram termo de consentimento pósesclarecido em duas vias.

Os procedimentos foram realizados por um médico radiologista e um médico cirurgião vascular, em conjunto. Os pacientes foram posicionados em decúbito dorsal com elevação do membro inferior tratado. O método de Tessari ${ }^{(\mathbf{1 6})}$ foi utilizado para a produção da espuma. No procedimento foram utilizadas duas seringas de $10 \mathrm{ml}$, scalp $\mathrm{n}^{\circ} 21$, agulhas $40 \times 12$ e um dispositivo de três vias para misturar a solução esclerosante ao ar. $\mathrm{O}$ agente esclerosante utilizado foi o polidocanol com concentração entre $1 \%$ e $3 \%$ e o volume total aplicado variou entre 5 e $20 \mathrm{ml}$ (média de 12,8 $\mathrm{ml}$ ).

A progressão da espuma dentro do vaso foi observada pelo ultrassom com Doppler colorido. Após o procedimento foi aplicada compressão elástica (com faixa tipo tensor) e os pacientes foram orientados a deambular por 15 a 20 minutos e manter a compressão elástica por três dias ininterruptos, e em seguida utilizar meia compressiva de 30 a $40 \mathrm{mmHg}$ por 20 a 30 dias.

Tabela 1 Resultado da ultrassonografia com Doppler colorido na primeira e segunda reavaliações no seguimento após o tratamento.

\begin{tabular}{lcccccc}
\hline & \multicolumn{2}{c}{ Primeira reavaliação } & & \multicolumn{2}{c}{ Segunda reavaliação } \\
\cline { 2 - 3 } \multicolumn{1}{c}{ Situação do vaso } & $n$ & $(\%)$ & & $n$ & $(\%)$ \\
\hline Oclusão total & 10 & $(55,6)$ & 4 & $(44,4)$ \\
Recanalização parcial & 5 & $(27,8)$ & 2 & $(22,2)$ \\
Recanalização total & 3 & $(16,7)$ & 3 & $(33,3)$ \\
Total & 18 & $(100)$ & 9 & $(100)$ \\
\hline
\end{tabular}

n, número de pacientes.
Os pacientes foram reavaliados pelo ultrassom com Doppler colorido após 7 dias e 15 dias, e o seguimento de longo prazo variou de 4 a 44 meses. As úlceras com reepitelização total e ausência de drenagem foram consideradas curadas. As varizes que apresentaram melhora clínica e oclusão total ou parcial da safena magna foram consideradas curadas. Foram considerados casos de recidiva quando ocorreu o rompimento epitelial da úlcera no membro tratado ou recidiva das veias varicosas com recanalização total da veia safena magna.

\section{RESULTADOS}

Os pacientes que participaram do estudo tinham em média 61 anos e $88,9 \%$ eram mulheres. A queixa principal foi úlcera venosa crônica (61\%), com tempo de duração entre 3 e 240 meses (média de 66 meses), seguida de varizes $(33,4 \%)$ e hipercromia $(5,6 \%)$. Entre as queixas secundárias observaram-se edema, dor, hiperemia e prurido. $\mathrm{O}$ membro inferior tratado foi o direito em $50 \%$ dos casos e apenas um paciente teve os dois membros tratados. Quanto ao número de aplicações, observou-se que maioria dos casos $(55,6 \%)$ recebeu apenas uma aplicação, 38,9\% receberam duas aplicações e um $(5,6 \%)$ paciente recebeu três aplicações. A Tabela 1 apresenta o resultado da ultrassonografia com Doppler colorido nas reavaliações após o procedimento.

A veia safena magna foi o vaso tratado em todos os casos. No primeiro retorno observou-se que $55,6 \%$ dos vasos mantinham oclusão total, $27,8 \%$ apresentavam recanalização parcial e 16,7\%, recanalização total com redução de diâmetro do vaso. O tempo de reavaliação foi, em média, de 13 meses, tendo variado de 1 a 37 meses. Nove pacientes foram avaliados duas ve- 
zes com ultrassom durante o seguimento. Nesse segundo retorno, $44,4 \%$ dos vasos tratados mantinham oclusão total, $22,2 \%$ apresentavam recanalização parcial e $33,3 \%$, recanalização total.

A avaliação clínica das varizes mostrou que entre os pacientes que apresentavam varizes como queixa principal (5 casos), todos apresentaram melhora clínica e observou-se um caso de recidiva, com recanalização total da veia safena magna. As demais $(80 \%)$ apresentaram melhora clínica e oclusão total ou recanalização parcial, com redução do diâmetro do vaso.

A avaliação dos pacientes que apresentavam úlceras venosas como queixa prin-

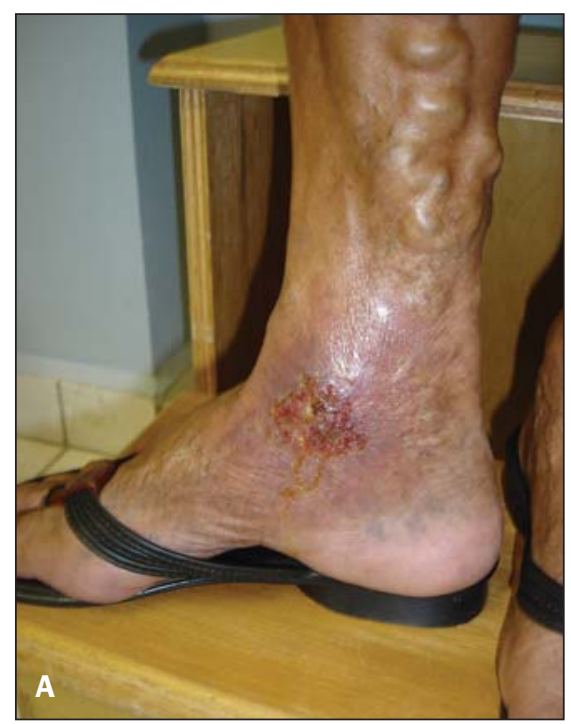

Figura 1. Membro inferior direito. A: Varizes e úlcera venosa crônica. B: Úlcera cicatrizada e resolução da veia varicosa após o procedimento.

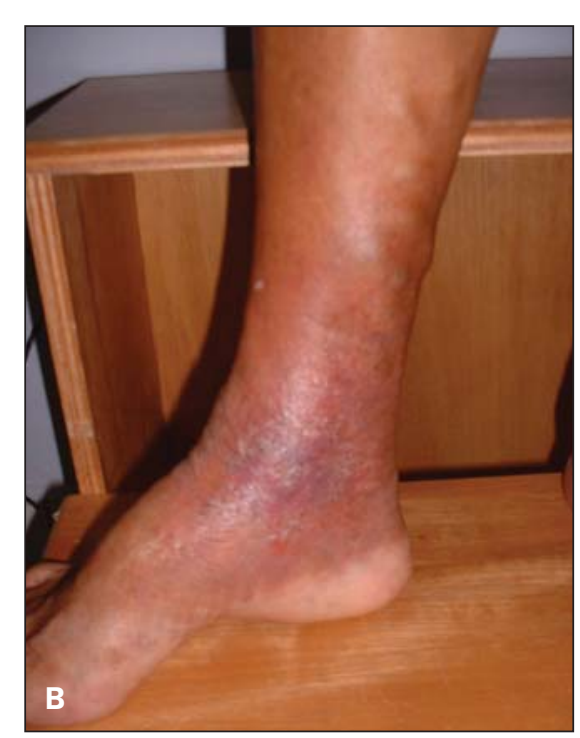

cipal (10 casos) mostrou que no primeiro retorno 7 estavam cicatrizadas, 2 apresentaram melhora mas não tiveram cicatrização completa e 1 cicatrizou mas apresentou recidiva. Assim, a taxa de cura das úlceras venosas foi de $70 \%$ e observou-se que $30 \%$ apresentaram recidiva ou melhora sem cicatrização completa.

A Figura 1 apresenta a evolução de um caso de varizes e úlcera no membro inferior direito. A Figura 2 mostra a evolução de um caso de úlcera venosa crônica. A Figura 3 apresenta um caso de varizes com veia safena magna dilatada e tortuosa antes do procedimento e o resultado 15 dias após o procedimento. A Figura 4 mostra duas ima每, gens de ultrassonografia: durante o procedimento e o controle um ano após.

A análise das complicações mostrou que $72,2 \%$ dos pacientes não apresentaram nenhuma complicação. Entre os casos que apresentaram algum evento adverso $(27,8 \%)$, observaram-se um caso de telangiectasia, três casos de coágulos superficiais e um caso de necrose superficial de pele. Todos os casos foram atendidos pelos médicos responsáveis pelo procedimento e resolvidos. A telangiectasia recebeu aplicações superficiais de agente esclerosante, os coágulos foram drenados com uma punção simples e compressão local e a necrose de pele foi debridada, tratada com curativo hidroativo e cicatrizou.

A avaliação da satisfação dos pacientes mostrou que $94,4 \%$ referiram estar satisfeitos com o resultado do tratamento, $88,9 \%$ afirmaram que repetiriam o tratamento se necessário e 77,8\% disseram que recomendariam o tratamento a um amigo.

\section{DISCUSSÃO}

Os resultados observados mostraram que a taxa de oclusão total ou recanalização parcial dos vasos tratados, que representa o sucesso do tratamento, foi de $83,4 \%$ na primeira revisão dos pacientes e de $66 \%$ entre os pacientes que tiveram uma segunda revisão. A taxa de remissão de varizes $(80 \%)$ e úlceras $(70 \%)$ foi muito boa e se assemelha aos resultados observados por outros autores.
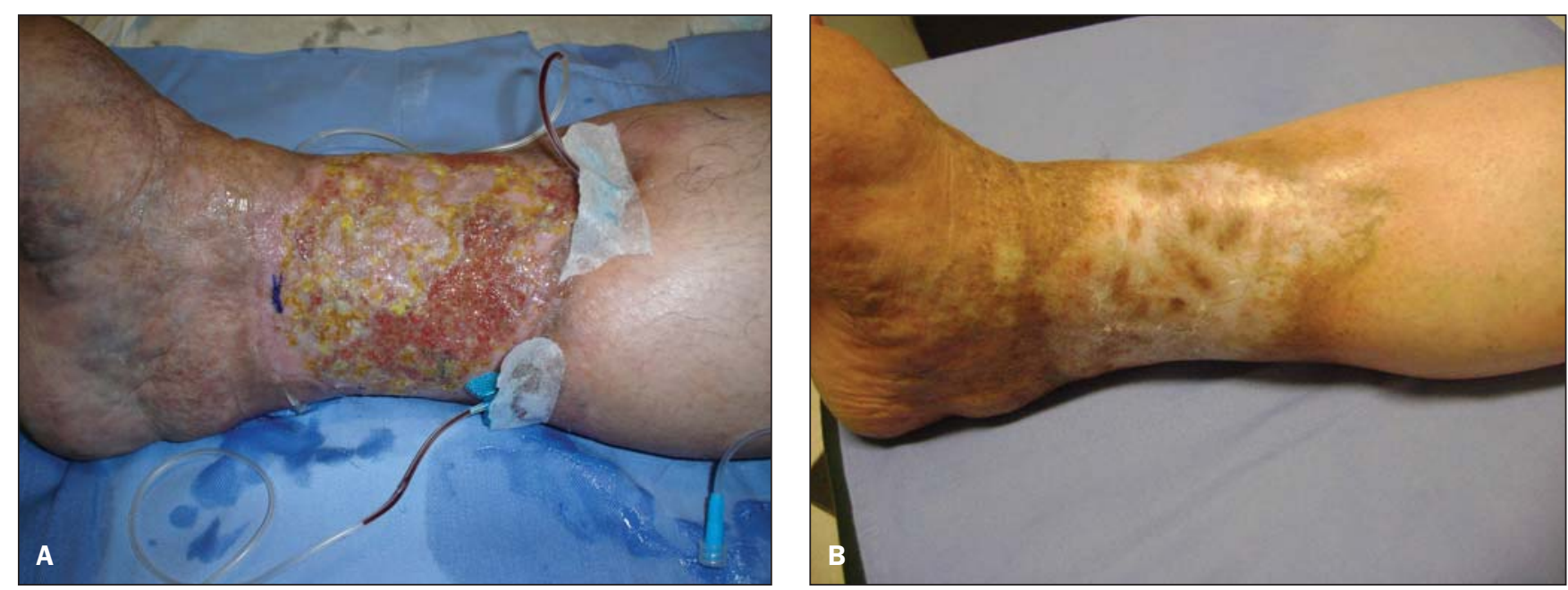

Figura 2. Úlcera venosa crônica. A: Úlcera venosa há 12 anos. Punção venosa para infusão do agente esclerosante. B: Úlcera cicatrizada. Aspecto da úlcera cicatrizada após o procedimento. 

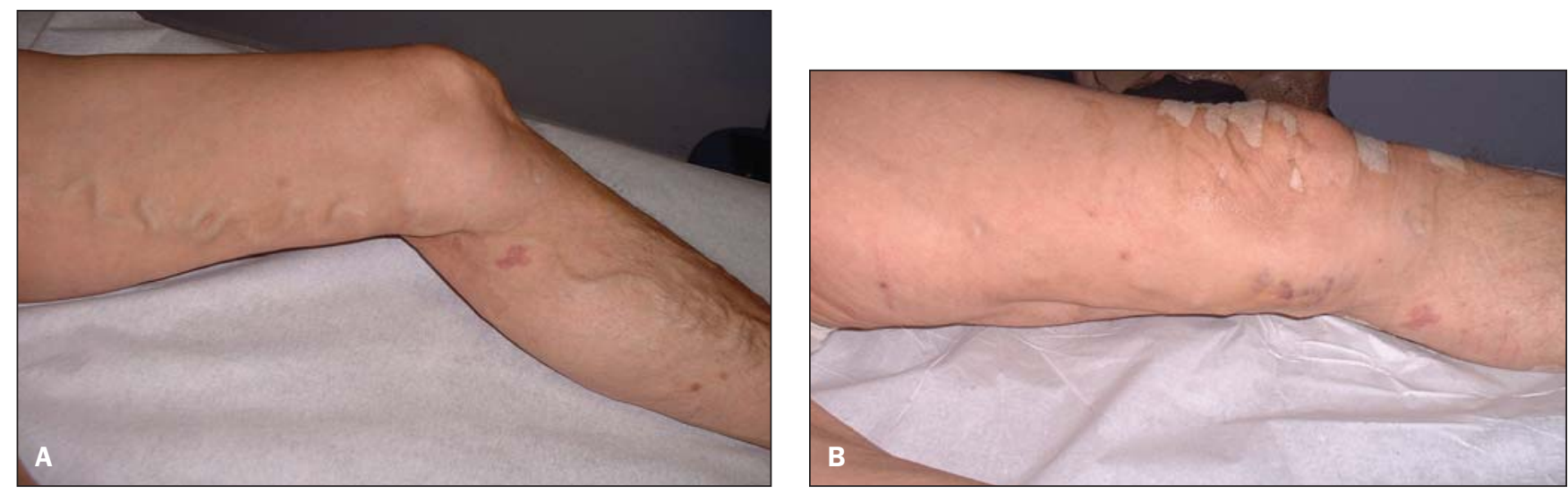

Figura 3. A: Membro inferior esquerdo antes do procedimento. Veia safena magna dilatada e tortuosa antes do procedimento. B: Membro inferior esquerdo após o procedimento. Resultado 15 dias após o procedimento. Veia safena magna completamente retraída.
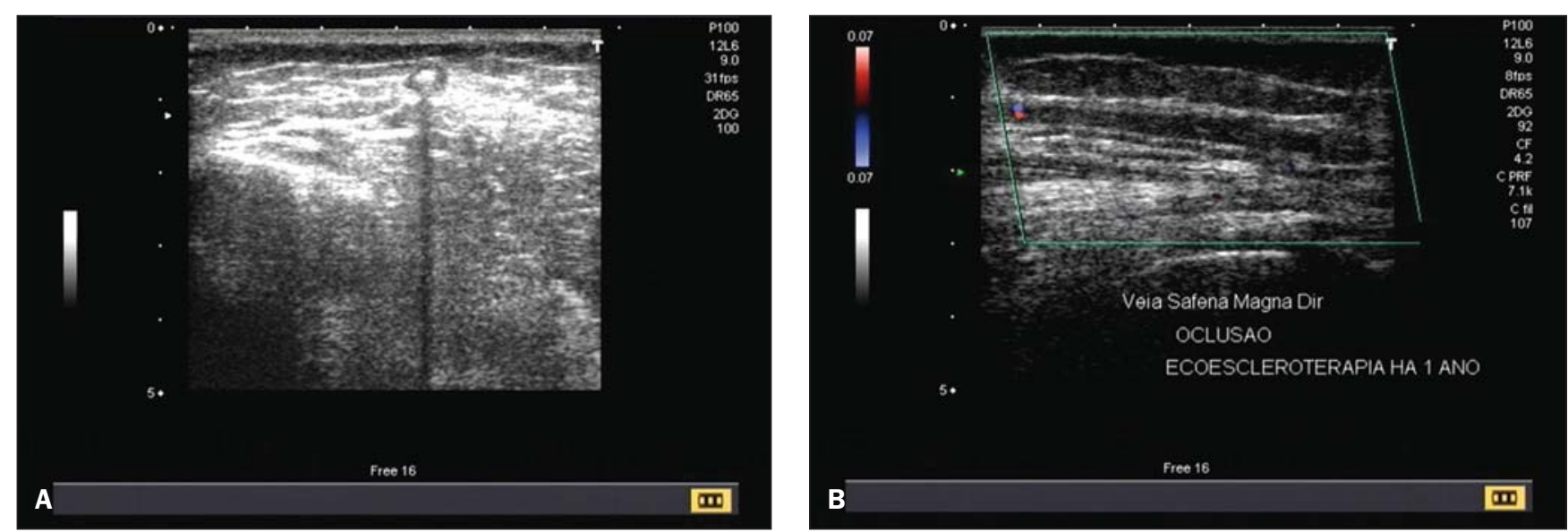

Figura 4. A: Ultrassonografia. Agente esclerosante ecogênico no interior da veia safena magna durante a aplicação. B: Ultrassonografia com Doppler colorido. Oclusão da veia safena magna direita, um ano após o procedimento.

Um estudo nacional que avaliou o tratamento de varizes por ecoescleroterapia com microespuma observou $84 \%$ de oclusão total e recanalização parcial ${ }^{(17)}$. Em outro estudo, que teve como objetivo descrever os resultados de um serviço de ecoescleroterapia com espuma, pesquisadores encontraram uma taxa de oclusão total de $74 \%$ e recanalização parcial de $10 \%$, seis meses após o tratamento ${ }^{(5)}$

Série clínica que apresentou os resultados de 459 membros com insuficiência venosa crônica relacionada à incompetência da veia safena magna tratados com ecoescleroterapia mostrou que $88 \%$ das veias permaneciam ocluídas seis meses ou mais após o tratamento. Os autores concluíram que esta técnica é útil no tratamento de insuficiência venosa crônica e pode ser considerada como uma alternativa à cirurgia ${ }^{(\mathbf{1 8 )}}$.

Pesquisa que estimou o sucesso da ecoescleroterapia com espuma no tratamento de doença venosa superficial crônica mostrou que a taxa de oclusão total ou ausência de refluxo em pacientes que receberam apenas uma aplicação foi de $52,4 \%$ no seguimento de 36 meses e de 76,8\% para os pacientes que receberam duas ou mais aplicações. Os pesquisadores concluíram que a ecoescleroterapia com espuma oferece resultados satisfatórios, considerando-se que o tratamento pode precisar ser repetido para se alcançar uma taxa de sucesso maior que $70 \%{ }^{(\mathbf{1 9})}$.

Estudos que compararam a ecoescleroterapia com outros métodos de tratamento para a insuficiência venosa crônica mostraram resultados contraditórios. Revisão Cochrane que comparou os resultados da cirurgia e da escleroterapia mostrou que a escleroterapia foi significativamente melhor que a cirurgia em um ano. No período de três a cinco anos após o tratamento, no entanto, a cirurgia mostrou melhores resul- tados. Os autores concluíram que não há evidências suficientes para recomendar o uso preferencial da cirurgia ou ecoescleroterapia para o tratamento de veias varico$\operatorname{sas}^{(20)}$.

Ensaio clínico randomizado que comparou pacientes com veias varicosas submetidos ao tratamento cirúrgico ou ecoescleroterapia com espuma mostrou que a veia safena magna permaneceu ocluída em $78 \%$ do grupo que recebeu cirurgia e em $90 \%$ dos pacientes que receberam a ecoescleroterapia com espuma 180 dias após o tratamento. Os autores concluíram que a ecoescleroterapia com espuma é uma opção de tratamento segura e efetiva para pacientes com insuficiência venosa crônica ${ }^{(21)}$.

Estudo que avaliou o custo-efetividade de vários tratamentos para veias varicosas mostrou que a ecoescleroterapia teve o menor custo inicial, mas precisou de mais intervenções do que os outros métodos. Para 
os autores desse estudo, a cirurgia ambulatorial e a ablação intravenosa a laser ou por radiofrequência foram os métodos de tratamento com melhor custo-efetividade ${ }^{(22)}$.

Há estudos mostrando que a ecoescleroterapia com espuma também apresenta ótimos resultados no tratamento de úlceras venosas crônicas. Estudo que avaliou a resposta de curto prazo ao tratamento de ecoescleroterapia com espuma (13 úlceras venosas crônicas em 9 pacientes) mostrou que em duas semanas 2 úlceras fecharam, 2 safenas magnas estavam ocluídas e houve melhora significativa das dimensões das demais úlceras e do diâmetro das safenas magnas tratadas. Os autores concluíram que pacientes com insuficiência venosa grave respondem rapidamente à ecoescleroterapia com espuma ${ }^{(23)}$.

Pesquisa recente que acompanhou 27 pacientes com úlceras venosas crônicas e teve como objetivo descrever a cura dessas úlceras e as taxas de recorrência após ecoescleroterapia com espuma mostrou que no seguimento de um mês $79 \%$ das úlceras venosas crônicas tinham sido curadas completamente e seis meses após o tratamento essa taxa de cura era de 96\%. Após 12 meses, $93 \%$ das úlceras permaneciam curadas e $7 \%$ apresentaram recorrência, que segundo os autores esteve relacionada à falta de compressão adequada $^{(24)}$.

Ensaio clínico randomizado que comparou a compressão $(n=20)$ e a ecoescleroterapia com espuma associada à compressão $(n=13)$ mostrou que em 12 semanas $62 \%$ das úlceras estavam curadas no grupo controle (compressão), em comparação a $92 \%$ de cura no grupo da ecoescleroterapia. Após 24 semanas observou-se $85 \%$ de cura no grupo controle e $92 \%$ no grupo ecoescleroterapia ${ }^{(25)}$.

As limitações do presente estudo foram a pequena casuística e amostra de conveniência, o que limita a generalização dos resultados. Além disso, os dados foram obtidos por meio do levantamento dos respectivos prontuários e da reavaliação dos pacientes tratados, ou seja, os resultados apresentados demonstram os resultados clínicos obtidos nos primeiros anos de utilização desta técnica, que tem sido pouco utilizada no Brasil, mas tem mostrado resultados animadores em inúmeras publicações internacionais.

Este estudo tem como ponto forte a descrição dos resultados da ecoescleroterapia com espuma, que pode ser utilizada como uma opção de tratamento para a insuficiência venosa crônica e tem apresentado bons índices de sucesso. Além disso, é uma técnica segura, rápida e com custos menores do que os da cirurgia, podendo ser uma opção para os pacientes que têm contraindicações ao procedimento cirúrgico.

\section{CONCLUSÕES}

A ecoescleroterapia mostrou-se um procedimento seguro e eficaz para o tratamento de insuficiência venosa crônica nesse grupo de pacientes. As complicações observadas foram mínimas e a maioria dos pacientes referiu satisfação com os resultados do tratamento.

\section{REFERÊNCIAS}

1. Redondo P, Cabrera J. Microfoam sclerotherapy. Semin Cutan Med Surg. 2005;24:175-83.

2. Morrison N. Ultrasound-guided foam sclerotherapy: safety and efficacy. Phlebology. 2009;24: 239.

3. Zimmet SE. Sclerotherapy treatment of telangiectasias and varicose veins. Tech Vasc Interv Radiol. 2003;6:116-20.

4. Guex JJ, Allaert FA, Gillet JL, et al. Immediate and midterm complications of sclerotherapy: report of a prospective multicenter registry of 12,173 sclerotherapy sessions. Dermatol Surg. 2005;31:123-8.

5. O'Hare JL, Parkin D, Vandenbroeck CP, et al. Mid term results of ultrasound guided foam sclerotherapy for complicated and uncomplicated varicose veins. Eur J Vasc Endovasc Surg. 2008; 36:109-13.

6. Morrison N, Neuhardt DL. Foam sclerotherapy: cardiac and cerebral monitoring. Phlebology. 2009;24:252-9.

7. Gillet JL, Guedes JM, Guex JJ, et al. Side-effects and complications of foam sclerotherapy of the great and small saphenous veins: a controlled multicentre prospective study including 1,025 patients. Phlebology. 2009;24:131-8.

8. Chapman-Smith P, Browne A. Prospective five- year study of ultrasound-guided foam sclerotherapy in the treatment of great saphenous vein reflux. Phlebology. 2009;24:183-8.

9. Pascarella L, Bergan JJ, Mekenas LV. Severe chronic venous insufficiency treated by foamed sclerosant. Ann Vasc Surg. 2006;20:83-91.

10. Orbach EJ. Sclerotherapy of varicose veins: utilization of intravenous air block. Am J Surg. 1944; 66:362-6.

11. Cabrera J, Cabrera García-Olmedo JR. Nuevo método de esclerosis en las varices tronculares. Patología Vascular. 1995;4:55-73.

12. Coleridge Smith P. Saphenous ablation: sclerosant or sclerofoam? Semin Vasc Surg. 2005;18:19-24.

13. Bastos FR, Lima AE, Assumpção AC. Ecoescleroterapia de varizes com espuma: revisão de literatura. Rev Méd Minas Gerais. 2009;19:38-43.

14. Bunke N, Brown K, Bergan J. Foam sclerotherapy: techniques and uses. Perspect Vasc Surg Endovasc Ther. 2009;21:91-3.

15. de Waard MM, der Kinderen DJ. Duplex ultrasonography-guided foam sclerotherapy of incompetent perforator veins in a patient with bilateral venous leg ulcers. Dermatol Surg. 2005;31:580 3.

16. Tessari L. Nouvelle technique d'obtention de la scléro-mousse. Phlébologie. 2000;53:129.

17. Figueiredo M, Araújo SP, Penha-Silva N. Ecoescleroterapia com microespuma em varizes tronculares primárias. J Vasc Bras. 2006;5:177-83.

18. Smith PC. Chronic venous disease treated by ultrasound guided foam sclerotherapy. Eur J Vasc Endovasc Surg. 2006;32:577-83.

19. Myers KA, Jolley D, Clough A, et al. Outcome of ultrasound-guided sclerotherapy for varicose veins: medium-term results assessed by ultrasound surveillance. Eur J Vasc Endovasc Surg. 2007;33:116-21

20. Rigby KA, Palfreyman SJ, Beverley C, et al. Surgery versus sclerotherapy for the treatment of varicose veins. Cochrane Database Syst Rev. 2004;(4):CD004980.

21. Figueiredo M, Araújo S, Barros N Jr, et al. Results of surgical treatment compared with ultrasound-guided foam sclerotherapy in patients with varicose veins: a prospective randomised study. Eur J Vasc Endovasc Surg. 2009;38:758-63.

22. Gohel MS, Epstein DM, Davies AH. Cost-effectiveness of traditional and endovenous treatments for varicose veins. Br J Surg. 2010;97:1815-23.

23. Hertzman PA, Owens R. Rapid healing of chronic venous ulcers following ultrasound-guided foam sclerotherapy. Phlebology. 2007;22:34-9.

24. Darvall KAL, Bate GR, Adam DJ, et al. Ultrasound-guided foam sclerotherapy for the treatment of chronic venous ulceration: a preliminary study. Eur J Vasc Endovasc Surg. 2009;38:7649.

25. O'Hare JL, Earnshaw JJ. Randomised clinical trial of foam sclerotherapy for patients with a venous leg ulcer. Eur J Vasc Endovasc Surg. 2010; 39:495-9. 